\title{
Sarcoma miofibroblástico posradioterapia
}

\author{
Radiation-induced myofibroblastic sarcoma
}

Mario Almeida Branco S ${ }^{1}$, José Luis Vargas $\mathrm{F}^{1}$, Francisco Fernández L'1.

\begin{abstract}
RESUMEN
Paciente de 51 años, varón, fumador y alcohólico moderado, con síntomas de disfonía intermitente que debuta con una adenopatía cervical metastásica de carcinoma epidermoide, sin hallazgos del tumor primario. Es intervenido quirúrgicamente siéndole realizada una disección cervical unilateral y posteriormente es tratado con quimiorradioterapia concomitante. A los 4 años del final del tratamiento oncológico, sin recidiva local ni aparición del tumor primario, el paciente debuta con disnea de presentación subaguda. Tras la exploración física y pruebas diagnósticas, se constata una nueva tumoración en laringe que resulta ser un "sarcoma miofibroblástico de bajo grado". Según los criterios de Cahan, este tumor, de estirpe histológica poco frecuente en laringe, hace pensar que probablemente podría ser inducido por la radiación. Se discuten los criterios de causalidad entre radiación y oncogénesis y se resumen las características de los tumores radioinducidos.
\end{abstract}

Palabras clave: Sarcoma radioinducido, radioterapia, laringe.

\begin{abstract}
We report a clinical case about a male patient, smoker and alcoholic, that debuts with a neck metastasis of an epidermoid carcinoma of unknown origin. He was submitted to a cervical dissection and treated with adjuvant combined chemo and radio therapy. Four years after finishing the oncologic treatment without tumor recurrence or appearence of the primary tumor, he begins with sub acute dyspnea. After physical examination and imaging, a new larynx tumor was found, "low grade myofibroblastic sarcoma". Under Cahan criteria, this tumoral kind, rare in larynx, was probably induced by the local radiotherapy. We discuss briefly the causality between radiation and oncogenesis and the characteristics of the radio induced tumors.
\end{abstract}

Key words: Radiotherapy, Radio-induced sarcoma, Larynx.

1 Médico Servicio de Otorrinolaringología. Hospital Universitario San Cecilio. Granada. España.

Recibido el 15 de marzo de 2015. Aceptado el 6 de septiembre de 2015. 


\section{INTRODUCIÓN}

La radioterapia es una modalidad de tratamiento usada con frecuencia en los tumores de cabeza y cuello, tanto para el tratamiento de los tumores primarios, como para los metastásicos. No obstante, este tipo de tratamiento no está exento de complicaciones. Los tumores radioinducidos son una de las complicaciones tardías más devastadoras del uso de la radioterapia. Los criterios de Cahan son actualmente la herramienta más utilizada para establecer esta relación de causalidad entre radiación y oncogénesis. Presentamos un caso poco frecuente de un paciente que debutó con metástasis cervicales de carcinoma epidermoide, de tumor primario desconocido. Fue tratado con cirugía y varios ciclos de radioterapia y como consecuencia del tratamiento desarrolló un tumor radioinducido en el área irradiada.

\section{OBJETIVO}

Presentar a la comunidad médica otorrinolaringológica nuestra experiencia con un caso de tumor radioinducido, así como la discusión de los aspectos específicos y de su manejo terapéutico.

\section{CASO CLÍNICO}

Presentamos el caso de un paciente varón de 51 años, ex-fumador de 20 paq/año y bebedor habitual moderado, sin otros antecedentes personales de interés. Acude por primera vez a consultas de ORL por disfonía intermitente de varios meses de evolución. Tras la exploración clínica, se constata una lesión leucoplásica milimétrica en cuerda vocal derecha. Se le practica biopsia mediante microcirugía de la laringe y el resultado fue negativo para células malignas. Cinco meses después, el paciente debuta con adenopatía sospechosa en área II-A1 izquierda. El examen físico completo, incluyendo nasofibroscopía, fue negativo para lesiones tumorales. En el TAC cérvico torácico solo se observó una adenopatía de $4 \mathrm{~cm}$ en nivel II-A clasificándolo como TxN2aMx. Se practicó una PAAF de la adenopatía cuyo resultado fue informado como "metástasis de carcinoma epidermoide".
A consecuencia de los resultados, se le realizó una disección cervical funcional izquierda concomitante con laringoscopía directa, tomando biopsias de distintos puntos de la glotis y supraglotis. El informe de anatomía patológica reveló metástasis de carcinoma epidermoide en 3 de las 4 adenopatías cervicales extirpadas y ausencia de células malignas en todas las biopsias laríngeas.

Se presentó en comité oncológico y se decidió tratamiento adyuvante a la disección cervical. El tratamiento consistió en quimiorradioterapia concomitante, con cisplatino $(100 \mathrm{mg} / \mathrm{m} 2$ IV cada 28 días $\times 3$ ciclos) y radioterapia cervical hasta 66 Gy (46 Gy a todo el volumen y 20 Gy en el lecho tumoral) durante 3 meses. Tras finalizar el tratamiento oncológico, se efectuó un seguimiento del paciente mediante controles bimestrales en consultas de ORL y oncología. Durante este período el paciente se mantuvo asintomático, sin signos de recidiva tumoral locorregional, ni aparición del tumor primario.

A los 3 años y 7 meses después del final del tratamiento oncológico, el paciente consultó en el servicio de urgencias por disnea moderada de instalación subaguda. A la exploración física se observó una tumoración glótica de aspecto polipoideo que reducía el lúmen laríngeo y causaba parálisis bilateral de cuerdas vocales. La palpación cervical fue anodina. Se realizó un PET-TAC que mostró una zona hipercaptante en glotis y subglotis. Ante la persistencia de la disnea se le realizó una traqueostomía con anestesia local y toma de biopsia del tumor laríngeo. El informe de anatomía patológica reveló la presencia de un sarcoma miofibroblástico de bajo grado. Posteriormente se le realizó laringectomía total con márgenes amplios y vaciamento cervical derecho con revisión del lado izquierdo (ver Figuras 1 y 2 de la pieza quirúrgica).

Tres meses después de la cirugía, se constató recidiva tumoral en la piel peritraqueostomal y lóbulo tiroideo izquierdo. El TAC de tórax no mostró otras lesiones. Se decidió una nueva intervención quirúrgica para exéresis de las lesiones en traqueostoma y la realización de hemitiroidectomía izquierda. La biopsia confirmó infiltración de las piezas extirpadas por sarcoma miofibroblástico.

A consecuencia de los hallazgos clínicos, se discute nuevamente el caso clínico con el servicio 


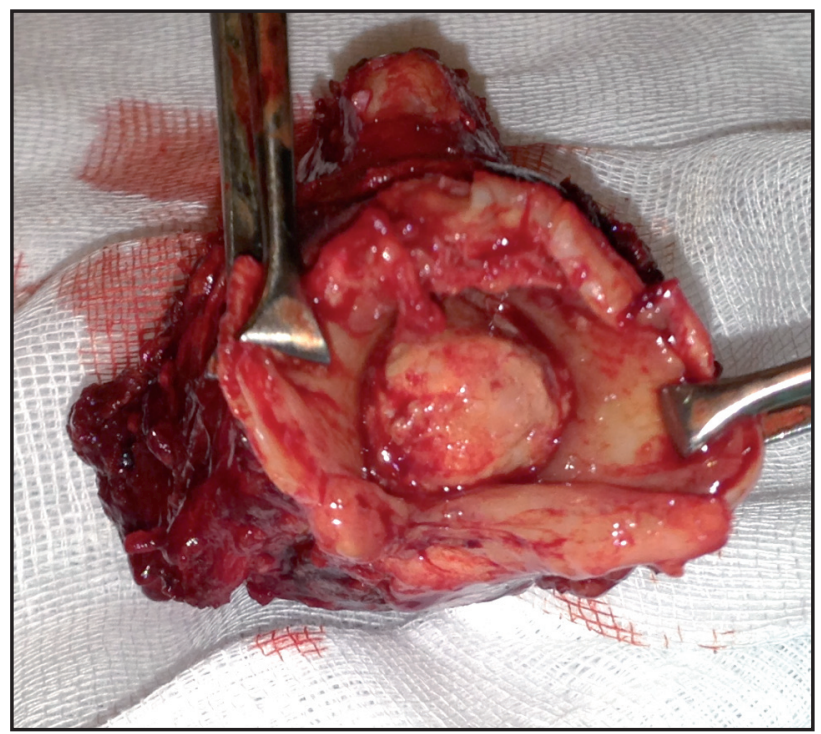

Figura 1. Imagen de la pieza quirúrgica de laringectomía total, donde se observa la disminución importante la luz glótica que motivó la traqueostomía.

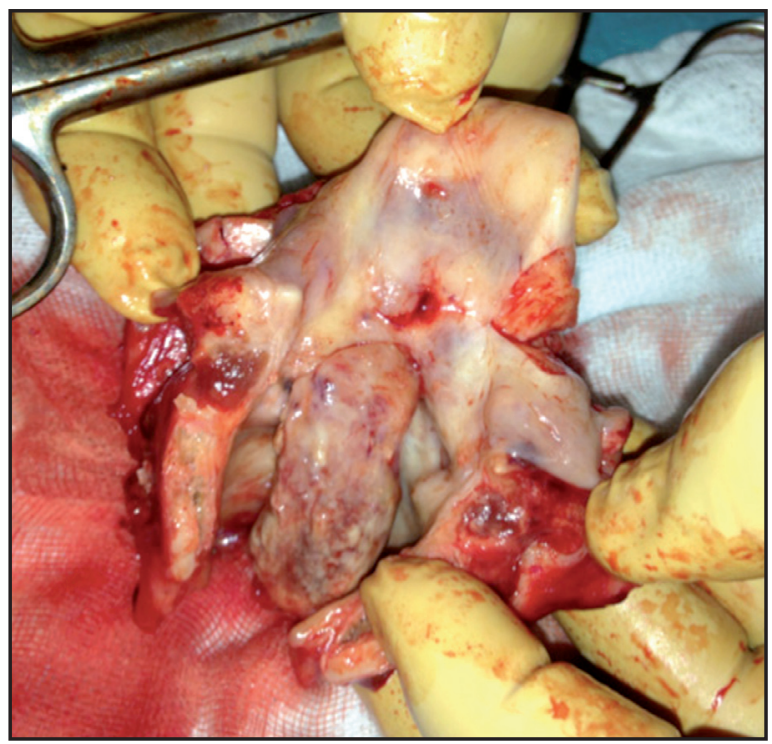

Figura 2. Imagen de la misma pieza quirúrgica. Note el aspecto fibroelástico y pediculado de la tumoración, que nace de la glotis.

de oncología y se decide realizar quimioterapia adyuvante con epirrubicina + ifosfamida en 6 ciclos. Asimismo se le realiza un seguimiento mediante PET-TAC en los que no se revela evidencia de recidiva tras 18 meses.

\section{DISCUSIÓN}

Nuestro paciente debutó con una metastásis cervical de carcinoma epidermoide de $4 \mathrm{~cm}$, clasificado como TxN2aMx. Tanto la localización, histología 
y estadio de nuestro caso, son los hallazgos que frecuentemente se encuentran en los tumores metastásicos de primarios desconocidos².

La radioterapia es una de las modalidades de tratamiento habitualmente usada para los carcinomas epidermoides de cabeza y cuello. Para las metástasis de primarios desconocidos es la primera opción de tratamiento adyuvante después de la cirugía o como terapia única en caso de tumores inoperables ${ }^{2}$. Sin embargo, la quimioterapia tiene un papel discutible 2 .

El tratamiento con radioterapia presenta complicaciones a corto y largo plazo. Una de las secuelas tardías más devastadoras son las neoplasias inducidas por radiación (NIR). Estas son neoplasias de cualquier estirpe embrionaria que se originan después de un tratamiento con radioterapia ${ }^{3,4}$. De todas ellas, las neoplasias de origen mesenquimal tipo sarcoma, son las más comunes ${ }^{3}$.

Los sarcomas inducidos por radiación (SIR), o sarcomas posradiación por la dificultad en establecer una relación causal, fueron descritos por primera vez en $1922^{4}$. Su incidencia estimada, aunque muy variable, se sitúa entre $0,4 \%-1 \% 3,5$, correspondiendo a $5,5 \%$ de todos los sarcomas ${ }^{6}$. No es de extrañar un aumento progresivo de nuevos casos conforme asistimos a una mayor supervivencia de los pacientes tratados con radioterapia.

Los criterios de Cahan modificados son todavía la herramienta más usada para sospechar de un tumor inducido por radiación 6 :

- El tumor se originó en un área previamente irradiada.

- El nuevo tumor fue histológicamente diferente del original.

- No existía evidencia del nuevo tumor al comienzo de la radioterapia.

- Existía latencia entre la irradiación y el desarrollo del nuevo tumor.

Nuestro paciente tuvo 4 de 4 de los criterios de Cahan modificados y la latencia de aparición fue de 3 años y 7 meses. La latencia de Ios SIR descrita en la bibliografía es muy amplia, pudiendo variar de meses a décadas ${ }^{3,6,7}$ y es inversamente proporcional a la dosis de radiación recibida ${ }^{5,8}$. Los sarcomas tienen menor latencia que otras $\mathrm{NIR}^{3}$.

La incidencia de SIR es dosis dependiente. Mientras que una dosis inferior a $10 \mathrm{G}$ es consi- derada despreciable, existe un enorme aumento de la incidencia con dosis superiores a $55 \mathrm{G}^{7-9}$. Asimismo, como ocurre en nuestro caso, el riesgo de aparición es mayor cuando se asocia quimioterapia concomitante ${ }^{6,7}$ y cuanto menor es la edad de la exposición inicial ${ }^{10}$.

Existen varios tipos histológicos de sarcoma y la mayoría son de alto grado ${ }^{10}$. Sus porcentajes varían mucho entre series, siendo los más comunes el osteosarcoma, fibrosarcoma, fibrohistiocitoma maligno, tumor inflamatorio miofibroblástico, angiosarcomas y leiomiosarcomas ${ }^{4,6,10}$. Estos sarcomas pueden aparecer en cualquier región del cuerpo de origen mesenquimal que haya sido irradiada y no tienen predilección por un lugar específico dentro del área ORL ${ }^{10}$. En nuestro caso, el sarcoma miofibroblástico de bajo grado, es una entidad poco frecuente, apareciendo con mayor frecuencia en la región de cabeza y cuell $0^{9}$, con un curso más indolente, pero con igual capacidad para producir recidivas locales ${ }^{4,11}$.

Esta variedad de sarcoma, se compone de miofibroblastos dispuestos en fascículos, que son células fusiformes que albergan características compartidas de células del músculo liso y fibroblastos ${ }^{5,9,11,12}$. Histológicamente la mayoría de las lesiones presentan un patrón infiltrativo difuso con moderada atipia nuclear ${ }^{9,11,12}$. Pueden ser de grado bajo, intermedio 0 alto $0^{8,9}$ y poseen marcadores positivos para células miogénicas ${ }^{5,9,11,12}$. No obstante no es fácil definir criterios histopatológicos que distingan los SIR de los sarcomas esporádicos ${ }^{10}$. Es posible que con la identificación de marcadores moleculares 0 genéticos se pueda llegar a algún consenso sobre esta temática ${ }^{10}$.

Asimismo, este tipo de sarcoma suele presentarse como una masa indolora de crecimiento progresiv $0^{12}$ con alta incidencia de recidiva local y de metástasis a distancia ${ }^{5}$. La técnica de imagen más sensible es la $\mathrm{RMN}^{7}$ y poseen patrones radiológicos característicos que los hacen distintos a los sarcomas primarios ${ }^{8}$.

El tratamiento de elección actual consiste en la extirpación con amplios márgenes quirúrgicos, por el limitado uso de radioterapia adicional y de la relativa insensibilidad a la quimioterapia ${ }^{4,10}$, los cuales no parecen aumentar la supervivencia ${ }^{6}$. La irradiación solo debe ser planteada cuando no existen otras alternativas y está dependiente de la 
dosis de radiación administrada previamente, así como de los órganos blanco a irradiar ${ }^{10}$.

En nuestro caso, la recidiva local probablemente se debiera a la extirpación con pocos márgenes quirúrgicos en la primera cirugía. En la segunda intervención fuimos más agresivos con los márgenes. Además el hecho de haber realizado una traqueostomía previa a la laringectomía total, puede haber sido causa de diseminación cutánea y tiroidea.

En relación a la expectativa de vida, los SIR suelen tener mal pronóstico, con tasas de supervivencia a los 2 años de $22 \%-48 \% 4,6$ y menor de $30 \%$ a los 5 años ${ }^{3,4,6}$, siendo incluso peores cuando son comparados con sarcomas esporádicos ${ }^{5,10}$. Los factores que reducen la supervivencia son la presencia de metástasis ${ }^{5,6}$, el lugar de aparición ${ }^{6}$, la resección sin márgenes quirúrgicos amplios ${ }^{6}$ y el tamaño tumoral ${ }^{4}$. El grado histológico de diferenciación no parece tener influencia en la agresividad tumoral ${ }^{4}$.

En cuanto al periodo de latencia, dada la variabilidad, es necesario un seguimiento a largo plazo, sobre todo de pacientes jóvenes.

\section{CONCLUSIÓN}

Una vez que un elevado número de pacientes recibirá radioterapia como componente habitual de su tratamiento oncológico en tumores de cabeza y cuello, es de especial importancia, un adecuado conocimiento de los tumores inducidos por radiación y un acertado manejo de este tipo de pacientes. Debido a la presencia de diferentes periodos de latencia entre la irradiación y el desarrollo del nuevo tumor, recomendamos la necesidad de un seguimiento largo e individualizado del paciente, siendo de especial importancia en pacientes jóvenes. Debido a la alta tasa de recidivas locales, la cirugía con extirpación tumoral con amplios márgenes, juega un papel importantísimo por la escasa respuesta a otro tipo de tratamientos.

El avance de las técnicas de biología molecular para el estudio de la genética de los SIR, serán fundamentales en un futuro. Cuando se consiga identificar y localizar los mecanismos responsables de la sarcomatogénesis, se podrá actuar sobre ellos, minimizando así los efectos de la radiación sobre los tejidos sanos.

\section{BIBLIOGRAFÍA}

1. Som PM, Curtin HD, Mancuso AA. An imagingbased classification for the cervical nodes designed as an adjunct to recent clinically based nodal classifications. Arch Otolaryngol Head Neck Surg 1999; 125: 388-96.

2. Calabrese L, Jereczek-Fosa, Jassem J, Rocca A, Bruschini R, Orecchia R, et al. Diagnosis and management of neck metastases from an unknown primary. Acta Otorrhinolaringologica Italiana 2005; 25: 2-12.

3. Sale K, Wallace D, Girod D, Tsue T. Radiationinduced malignancies of the head and neck. Otolaryngol Head Neck Surg 2004; 131: 643-6.

4. Lagrange Jl, Ramaioli a, Chateau MC, Marchal C, Resbeut M, Richaud P, Lagarde P, Rambert P, Tortechaux J, Seng SH, de la Fontan B, Reme-Saumon M, Bof J, Ghnassia JP, Coindre JM. Sarcoma after radiation therapy: retrospective multiinstitutional study of 80 histologically confirmed cases. Radiology 2000; 216: 197-205.

5. Korampalli TS, Mathew B, Strafford nd. Post radiation myofibrosarcoma of hypopharynx. $J$ Surg Case Rep 2013; rjs049.

6. Mavrogenis AF, Stavropoulos NA, Angelini A, Papagelopoulos PJ, Ruggieri P. Post radiation sarcomas. Acta Orthopaedicaet Traumatologica Hellenica 2012; 63: 161-5.

7. King AD, Ahuja AT, Teo P, Tse GM, Kew J. Radiation induced sarcomas of the head and neck following radiotherapy for nasopharyngeal carcinoma. Clin Radiol 2000; 55: 684-9.

8. ShePpARd DG, LibSHItz HI. Post-radiation sarcomas: a review of the clinical and imaging features in 63 cases. Clin Radiol 2001; 56: 22-9.

9. Tomohiro Y, Tomohide Y, Nadya K, Sasabe E, Ohno S, Үамамото T. Low-grade myofibroblastic sarcoma of the palate. Int J Oral Sci 2012; 4: 170-3.

10. Thiagarajan A, Iyer NG. Radiation-Induced sarcomas of head and neck. World J Clin Onco 2014; 5: 973-81.

11. Mentzel T. Myofibroblastic sarcomas: a brief review of sarcomas showing a myofibroblastic 
line of differentiation and discussion of the differential diagnosis. Current Diagnosis Pathology 2001; 7: 17-24.

12. Mentzel T, Dry S, Katenkamp D, Fletcher C. Low-
Grade myofibroblastic Sarcoma: Analysis of 18 Cases in the Spectrum of Myofibroblastic Tumors. American J Surg Pathology 1998; 22: 1228-38. 